\title{
La vie religieuse des Acadiens à travers leurs croyances traditionnelles
}

\section{Catherine Jolicoeur}

Volume 48, 1981

URI : https://id.erudit.org/iderudit/1007102ar

DOI : https://doi.org/10.7202/1007102ar

Aller au sommaire du numéro

Éditeur(s)

Les Éditions Historia Ecclesiæ Catholicæ Canadensis Inc.

ISSN

0318-6172 (imprimé)

1927-7067 (numérique)

Découvrir la revue

Citer cet article

Jolicoeur, C. (1981). La vie religieuse des Acadiens à travers leurs croyances traditionnelles. Sessions d'étude - Société canadienne d'histoire de l'Église catholique, 48, 79-88. https://doi.org/10.7202/1007102ar

Tous droits réservés @ Les Éditions Historia Ecclesiæ Catholicæ Canadensis Inc., 1981
Ce document est protégé par la loi sur le droit d'auteur. L'utilisation des services d'Érudit (y compris la reproduction) est assujettie à sa politique d'utilisation que vous pouvez consulter en ligne.

https://apropos.erudit.org/fr/usagers/politique-dutilisation/ 


\section{La vie religieuse des Acadiens à travers leurs croyances traditionnelles *}

La définition classique du mot légende, venant du latin legenda, signifie ce qui doit être lu. Mais la définition traditionnelle considère la légende comme le lien vivant entre le passé et le présent, le reflet de la vie et de la pensée. Elle serait le récit de la croyance ou la croyance qui devient le prétexte de la création du récit. De plus, elle porte en elle une leçon salutaire. Elle nous permet ainsi de découvrir la mentalité d'un peuple et même la pensée des lointains ancêtres.

Mais avant de nous plonger dans les légendes acadiennes, si nous jetions un regard sur l'époque précédent l'établissement des Acadiens du Nouveau-Monde, nous pourrions découvrir d'intéressants détails sur la vie religieuse des Acadiens d'autrefois et sur leurs légendes. Un bon nombre d'Acadiens, originaires du Poitou, sont de descendance celtique ; " et les Celtes, qui paraissent avoir été les premiers à quitter la patrie, possédaient déjà un fond de connaissances, de croyances et de coutumes dont nous retrouvons encore des traces. De plus, nous pouvons déceler dans leurs croyances des restes de paganisme romain, des souvenirs de la mythologie germanique ' ", et des coutumes de toutes espèces. Le peuple tient à ces croyances du passé malgré les efforts du clergé et des philosophes pour faire disparaître les superstitions.

L'un des plus remarquables prélats, Mgr Étienne Le Camus, évêque de Grenoble (1671-1707), avait pour but de redresser les moeurs des curés et des paroissiens et d'extirper le scandale et les superstitions. Son diocèse comptait environ trois cents paroisses ; il a fallu dix gros registres pour contenir les comptes rendus des visites pastorales de $\mathrm{M}^{\mathrm{r}}$ Le Camus.

Celui-ci interdisait constamment la danse, activité diabolique que certains prêtres favorisaient. Cette interdiction revient quarante-deux fois

1 Léon Pineau. Le Folk-Lore du Poitou. Paris, Leroux, 1892, vii. 
dans un extrait de 257 comptes rendus des visites de 1672 à 1705 : danses et batailles à éviter sous peine d'excommunication encourue ipso facto. Afin de prévenir ces actes, les prêtres devaient fermer les portes de l'église après avoir célébré la messe à quatre heures du matin, lorsqu'il y avait des fêtes où il s'était produit du désordre et du scandale dans le passé.

L'évêque se rend compte aussi de la confiance intéressée des paroissiens envers leur curé. Lors d'une tempëte où la grële avat endommagé blés et vignes, le peuple en colère reproche au prêtre son absence et le rend coupable du mal causé par la grêle. Mais, déclare l'évêque, ce sont leurs péchés qui attirent ces sortes de punitions publiques ; et l'idée qu'ils ont de la puissance du prêtre rend ces curés esclaves de leurs paroissiens.

Cependant $\mathrm{M}^{\mathrm{gr}}$ autorise un curé à faire l'exorcisme des rats qui mangent les blés ; parce que, dit-il, le peuple a une grande foi aux prières de l'Église et ils sont persuadés que la disparition des rats se produit aussitôt après les prières récitées par le prêtre. Par prudence, il ajoute qu'il permet l'exorcisme en attendant qu'il ait le temps d'examiner à fond ces prières du rituel, ce petit livre dont il ne possède qu'un exemplaire publié en 1479 .

Lors d'une de ses dernières visites, $\mathrm{M}^{\mathrm{gr}}$ Le Camus doit partir à pied à cause de la descente assez rude. Il passe par la fondrière du diable, une pièce de terre écroulée dont le propriétaire, ne pouvant ni en être payé ni la vendre, l'avait donnée au diable. Et, d'ajouter le prélat, " je ne sais si c'est une histoire ou un conte, mais il est certain qu'il arrive souvent en ce pays de pareils accidents ${ }^{2}{ }^{\prime}$.

Ces documents sont incomplets pour notre étude puisqu'ils donnent peu de détails positifs de la vic religicuse des ancêtres. Ils conticnnent cependant des éléments que nous retrouvons dans les légendes acadiennes, comme l'interdiction des danses, des querelles, la communication avec le diable, la puissance du prêtre et la foi en lui, la punition divine et la justice immanente. Nous pouvons être assurés que les légendes françaises comptent un grand nombre de faits positifs tout comme celles des Acadiens. Comme le disait d'un air poétique l'écrivain Jean Defrasne : " Avec le peuple de l'Antiquité, les nations étrangères, les époques

2 Robert Chanaud, "Folklore et religion dans le diocèse de Grenoble à la fin du XVII siècle : les visites pastorales de Mgr Le Camus ", dans Le Monde Alpin et Rhodanien, no 1-4 (1977) 60-103. 
typiques, chaque province de France apporte sa contribution à cette grande enquête au pays du rêve, du fantastique, du merveilleux. Ainsi le folklore français apparaît-il comme synthèse de toutes ces traditions des petites patries qui s' intègrent dans la grande ${ }^{3}$ ".

Revenons maintenant en Acadie pour y retrouver quelques aspects de la vie religieuse des Acadiens d'autrefois. Nous commençons d'abord par l'intervention continue de Dieu, intervention concrétisée par le prêtre qui communique sa propre expérience en ce domaine et celle qu'il a puisée dans les livres. Nous constaterons ensuite les actes qui en découlent et les conséquences qui s'ensuivent.

Au début, les prêtres étaient surtout des missionnaires qui avaient de grandes étapes à parcourir. En leur absence, un homme préside aux offices religieux, comme les messes blanches, i.e. les prières et les chants liturgiques de la messe, les baptêmes et les sépultures. Le missionnaire complète ensuite certains actes et documents lors de son passage. Lorsque celui-ci se présentait, les gens se rendaient à un endroit central, ce qui obligeait les plus éloignés à parcourir de longues distances. En certains cas, le trajet durait quelques jours pour l'aller et le retour. Certains d'entre eux emmenaient une vache pour servir du lait frais aux enfants surtout.

Devant ces gestes quasi héroïques, les soucis religieux du prêtre étaient souvent éclipsés par l'admiration pour ces chrétiens avides de communiquer avec Dieu et son représentant. Plus tard, quand les paroisses commencent à s'établir, on a vu percer des inquiétudes de part et d'autre. On avait sans doute plus de loisirs et de rapprochements pour constater les faiblesses des autres et leurs comportements quelquefois plus ou moins compromettants. C'est alors que se découvrent et s'accumulent des caractéristiques presque inexistantes auparavant : comme le rigorisme, la pudeur exagérée surtout chez les femmes, un certain fatalisme qui, déjà, avait commencé à faire partie de la philosophie des Acadiens. Et, comme le dit la folkloriste Lauraine Léger, " quelques croyances teintées de superstitions " qu'elle attribue à " l'omniprésence du sacré " chez le peuple qui "préfere expliquer par une cause surnaturelle ou pseudo-surnaturelle tout événement trop étranger ${ }^{4} »$.

3 M.-Louise et Jean Defrasne, Contes et légendes du Berry, Paris, Nathan, 1957, 5.

4 Lauraine LÉGER, Les sanctions populaires en Acadie, Montréal, Leméac, 1978, 122 . 
C'est alors que surgissent les grands péchés et leurs châtiments : comme l'empêchement de famille, la boisson alcoolique. la danse, la sorcellerie, les hommes vendus au diable et autres. Et presque tout événement pénible, douloureux ou mortel était attribué à une faute commise par la victime ou par les siens.

L'empêchement de la famille était une décision des époux de mettre fin à la procréation d'enfants : et quelle que soit la raison qui motivait

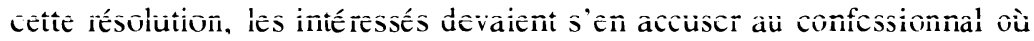
ils recevaient une semonce appropriée. Une grand-mère du Maine. E.E.. raconte à l'informateur. son petit-fils, un fait réel qui s"est produit au Maine dans le temps de sa mère, soit vers le début du XIX ${ }^{\mathfrak{c}}$ siècle. Un jour d'hiver. un couple volontairement sans enfant. a vu arriver une carriole ct les trois personnes dans la voiture étaient un prêtre et deux religieuses. Ceux-ci sont entrés dans la maison. Alors trois voix se font entendre l'une après l'autre: "Je devais naître et on n'a pas voulu me laisser naître ". (ter). L'homme et la femme échangent un regard et quand ils se retournent vers les visiteurs. il n'y a plus rien. tout est disparu.

Pour ce qui est de l'alcoolisme, ce fléau était condamné du haut de la chaire. Et si les buveurs mouraient en été d'ébriété, ils étaient enterrés hors du cimetière tout comme les enfants morts sans baptême, les personnes qui ne faisaient pas leurs pâques, les adultères et ceux qui ne payaient pas leur dîme. Ceux qui s'adonnaient au commerce de la boisson étaient menacés des pires malheurs. Il arrivait souvent qu'ils perdaient leurs biens dans un incendie, une administration défectueuse ou autre. D'où le proverbe populaire : " Farine du diable retourne en son".

La danse interdite avait aussi ses sanctions ; cette faute qu'on devait accuser privait le pénitent de l'absolution, à moins qu'il ne promette de ne plus danser. Il va sans dire qu'au temps des pâques, les promesses étaient un peu douteuses en certains cas. Les danses interdites sont l'un des thèmes les plus populaires de la légende acadienne. Les nombreux témoigriages recueillis en Acadie et ceux des autres provinces et même en Europe possèdent des éléments communs : $1^{\circ}$ Une danseuse désobéit au prêtre et à ses parents. $2^{\circ}$ Le diable, en beau jeune homme, danse avec elle. $3^{\circ}$ On envoie chercher le prêtre qui le chasse. $4^{\circ}$ Le diable brise un objet qu'on n'a jamais pu réparer.

La légende qui suit m'a été racontée comme étant authentique. Vers 1825 , on a organisé une danse dans un petit village du comté de 
Restigouche, le Jeudi saint à minuit. Un beau jeune homme, vêtu comme un prince, bel habit et gants noirs, est entré. Il s'est mis à danser et quand il passait près du berceau, le bébé se " désâmait " à pleurer. La danseuse, elle, se sentait brûler là où le danseur posait les mains. Il la griffait. Le "violonneux ", apercevant les pieds de cheval du danseur envoie chercher le prêtre. Il brise son instrument et n'a plus joué du violon depuis ce temps. En même temps, un homme s'était pendu sous l'échelle qui servait d'escalier parce qu'il venait de découvrir l'infidélité de sa femme. Le prêtre est venu et il a chassé le diable danseur avec son fouet bénit et de l'eau bénite. En partant, le diable a décollé le côté de la maison et on n'a jamais pu le réparer. Cette maison s'est enfouie graduellement dans la terre et elle est complètement disparue il n'y a pas longtemps.

La sorcellerie, qui existait surtout au sud du Nouveau-Brunswick, provenait selon certains informateurs, d'un ponchon (baril) que les vagues avaient déposé sur le rivage dans la région de Memramcook. Il contenait des livres que l'on s'est hâté de distribuer à ceux qui savaient lire. C'était des livres de sorcellerie, le Petit Albert, qui ont causé beaucoup de soucis aux prêtres et de malheurs aux paroissiens. Les premiers s'évertuaient à le faire disparaître et les habitants étaient constamment menacés de sorts et de diableries. Il y en a même qui allaient se vendre au diable. On raconte qu'une femme avait signé de son sang le nom de son mari ; et le diable en a été satisfait puisqu'il est apparu en boule de feu à son nouveau client.

Le diable est le plus populaire des êtres fantastiques dans les légendes acadiennes. Lui-même se métamorphosait en animal tout comme les sorciers et leurs adeptes ; et il s'associait aussi aux loups-garous. Les loupsgarous étaient des chrétiens qui, après avoir été sept ans sans accomplir leur devoir pascal, prenaient la forme d'un animal. En Acadie, c'était surtout la forme d'un gros chien noir qu'il prenait ; puis il agaçait les gens pour se faire délivrer. Pour y arriver, il fallait le blesser de façon à ce que le sang coule.

Bien que les Acadiens aient été remarquablement honnêtes, certaines exceptions démontraient la faiblesse humaine de quelques-uns ; et d'inoubliables punitions mettaient sans doute fin aux larcins. Une dame racontait qu'un homme avait volé une loutre prise dans le piège de son oncle. Celui-ci va en prévenir le curé qui se rend chez le voleur pour lui faire remettre l'animal à son propriétaire ; mais il est accueilli par un refus. Quelque temps après, la femme du voleur accouche d'un bébé qui ressemble à une loutre. La sage-femme, qui était la grand-mère de 
l'informatrice, suggère d'appeler le prêtre. Celui-ci se rend chez le voleur et aperçoit ce bébé-loutre qui se traîne sur le lit. Il dit au père de le déposer dans une boîte et de le placer dans l'église. Le bébé meurt durant la nuit et on l'enterre le lendemain. Ceci s'est produit vers 1900.

Les prêtres de cette époque avaient des responsabilités variées. En plus de leurs tâches sacerdotales, ils devaient se faire avocats, juges, notaires, médecins, car ces services n'existaient pas encore dans les villages. Les paroissiens avaient, en général, une grande confiance en leur prêtre et recouraient à lui dans leurs épreuves de toutes sortes. Par exemple, lors d'un incendie, le curé venait prêter son concours et parfois, obtenir un miracle qui éteignait le feu par la prière, les objets religieux, la pluie commandée, le vent qui tourne et bien d'autres. Les miracles de ce genre à Saint-Quentin, N.-B., où le prêtre a fait jaillir de l'eau d'un endroit où il n'en existait pas et a fait tourner le vent, sont attribués au curé, $\mathbf{M}^{\mathrm{r}}$ Martin, et au vicaire qui est actuellement l'évêque d'Ottawa.

Considérant le prêtre comme celui qui "remplace le bon Dieu ", les Acadiens lui confient leurs malades que. parfois, seul un miracle peut guérir. Nous avons recueilli des centaines de témoignages à ce sujet. Après le récit d'un miracle, une informatrice, née en 1895, a voulu émettre une comparaison entre les prêtres de son temps et ceux d'aujourd'hui, tout en sauvegardant le principe qu'on ne doit jamais dire du mal d'un prêtre.

Si on voyait un de nos prêtres (actuel) faire de quoi de même, on aurait une terrible confiance en lui. (Autrefois) ils les prenaient pour des saints. Ils étaient des saints aussi. Leurs prêtres étaient plus saints que le monde d'aujourd'hui, je crois ; parce que les prêtres d'aujourd'hui, ils vivent plus dans le monde. Ils sont peut-être aussi saints d'une manière, peut-être dans leur esprit. Mais, d'un côté, ils voyiont tant d'affaires puis i travelliont plus dans le monde que les vieux prêtres. Les vieux prêtres, ils travailliont pas comme ça. Ils faisiont tout le temps pénitence. Ils aviont beaucoup de pouvoirs aussi.

Certaines légendes à propos du pouvoir des prêtres semblaient sortir de la bible. Par exemple, la guérison de la fille de Jaïre, dans Marc, 5, 21-43, ressemble à celle qui s'est produite à Lorne, N.-B.

Une fille était malade depuis longtemps et un soir, son père est allé avertir le prêtre que sa fille était mourante.

- J'irai demain, dit le prêtre.

- Demain, il sera trop tard, répond le père. Elle sera morte. 
Le lendemain matin, le père de la fille est revenu au presbytère pour dire au curé :

- Vous avez pas voulu venir hier soir, il est trop tard. Ma fille est morte.

- Elle est morte hier soir, dit le curé.

Il prend son livre de prières et s'en va à la maison de la défunte. Il se met à marcher sur le plancher et à prier. C'est supposé qu'il a tellement prié que la sueur coulait sur lui. Avant de partir, le prêtre a vu la fille ouvrir les yeux et parler. Ça m'a été conté pour une pure vérité. Elle a vécu assez vieille, cette femme-là. Ça c'était vrai. Il l'avait ramenée pour longtemps.

Une manifestation fantastique, celle des revenants, nous communiquera divers détails au sujet de la tradition religieuse des Acadiens. Comme ces derniers ont un souci scrupuleux d'honnêteté, ceux qui mouraient sans avoir payé une dette ou remis un objet emprunté revenaient pour payer ou, s'ils ne le pouvaient pas, demander au propriétaire de leur donner cette dette, i.e. en faire la remise.

Dans un autre cas, deux bûcherons, dont l'un ne croit pas à l'enfer, se promettent mutuellement que le premier à mourir viendrait dire à l'autre si l'enfer existe ou non. Quelque temps après la mort de celui qui ne croyait pas, son compagnon part un dimanche matin pour la chasse. Il entend des branches craquer en arrière de lui et, en se retournant, il aperçoit son ami défunt qui lui dit : "Oui, y a un enfer " ! En entendant ces mots, l'autre s'empresse d'aller à la messe et il y a été fidèle jusqu'à sa mort. Un autre rappel, moins fantastique, prend la forme d'une ritournelle qui rappelle les négligents à leurs devoirs religieux : "L'enfer est au mitan de la terre. C'est là que le diable à sept têtes nous attend avec sa grande fourche, si on fait pas notre prière ».

Renommés pour leur probité, les Acadiens sont aussi doués d'une grande délicatesse parfois cachée sous un aspect rude, surtout chez les hommes. Par exemple, en 1959, cinquante-cinq pêcheurs ont péri en mer lors d'un désastre de Baie Sainte-Anne, dans le comté de Northumberland. Un de ceux qui avaient échappé par miracle se sent coupable d'être encore vivant. Il y avait aussi ce vieillard moribond qui attendait l'arrivée du prêtre. On était à la fonte des neiges et les routes n'étaient pas sûres. Alors, il priait ainsi : "Seigneur Jésus, descendez en raquettes, les chemins sont tendres ".

La foi des Acadiens est même émouvante en certains cas. Comme celle d'un autre vieillard, né en 1889 , qui avait perdu quatre des plus chers de ceux qu'il aimait : deux fils de 17 et 20 ans, perdus en mer, 
et deux petits-enfants, ses préférés. écrasés par une automobile trop rapide. Il me disait : " $C^{\prime}$ 'est ma foi qui m`a aidé à supporter tout cela. Si t'as la foi gros comme une tête d'épingle. tu peux trancher les montagnes ".

En terminant, je voudrais donner quelques exemples de la vie de prière de nos ancêtres. Les coutumes, à l'église, étaient de faire le chemin de la croix avant la messe et réciter le chapelet pendant la messe. Les sermons. qui le plus souvent étaient très longs. permettaient aux hommes de se payer un bon somme et aux femmes, leurs épouses, de tenir les enfants tranquilles et leur mari réveillé.

À la maison, la prière en famille avait lieu chaque soir et au complet malgré les efforts des jeunes pour l'éviter ; car la prière était trop longue à leur avis et leur faisait perdre du temps. Les veillées étaient trop courtes, elles, car il fallait se retirer quand le maître de la maison montrait par quelques gestes et paroles qu'il était temps de partir. Quelques légendes racontent les punitions de ceux qui s'esquivaient et, à leur retour, se contentaient de marmotter un Ave endormi en se jetant au lit. Ils voyaient des bêtes effrayantes, jamais vues. Ces animaux fantastiques les poursuivaient jusqu'à la barrière que la peur leur faisait franchir d'un éclair. D'autres voyaient les revenants de quelque parent décédé qui leur reprochaient leur vie désordonnée. Il y en a même qui voyaient le diable et entendaient son ricanement victorieux. Cependant les apparitions diaboliques étaient assez rares quand il s'agissait des jeunes gens qui manquaient la prière en famille. Et, comme le disait une informatrice : "Dans ce temps-là, les gens priaient beaucoup. La vieille grand-mère a dû prier fort assez pour faire venir le diable ».

En plus des prières à Dieu et à son Fils, il y avait aussi des saints particuliers à qui l'on s'adressait pour obtenir des grâces spéciales : saint Antoine pour les objets perdus, saint Benoît dont la médaille guérissait les animaux malades; sainte Anne, elle, guérissait les humains ; saint Joseph était le patron de la bonne mort. Une informatrice raconte qu'elle veillait un homme âgé et mourant qui avait une grande dévotion à saint Joseph. Un matin, elle entend frapper à la porte et va ouvrir. Elle se trouve en face d'un vieillard à grande barbe blanche et lui demande ce qu'il veut. "Une tasse de thé ", dit-il. Elle lui offre d'autres aliments mais il refuse. Après avoir bu le thé, il remercie et s'en va. " J'étais curieuse, dit-elle, et j'ai regardé à la fenêtre pour voir de quel côté il allait. Je n'ai vu personne. Il avait disparu. Je trouve qu'il ressemblait beaucoup au Saint Joseph qu'il y avait dans un cadre au salon. Le vieux est mort peu de temps après. J'ai toujours pensé que c'était saint Joseph qui était venu le chercher ". 
Les Acadiens avaient une prédilection pour la Vierge Marie qu'ils considéraient comme leur Mère. Ils obtenaient d'elle des miracles et des faveurs de toutes sortes. Étoile de la mer, elle protégeait les marins ; et plusieurs informateurs racontent les miraculeux sauvetages dont ils ont été les témoins ou les bénéficiaires. Quand les bateaux passaient près d'un endroit où se trouvait une statue, les marins déployaient une voile ou abaissaient un mât pour saluer Marie.

Les pêcheurs avaient coutume de réciter, en latin les litanies de la Vierge, quand ils partaient pour la pêche. Femmes et enfants, sur le rivage, répondaient aussi longtemps qu'ils entendaient les hommes. Le journal The Yarmouth Herald du 21 juillet 1859, raconte ce qui s'est produit quelques années auparavant. Un matin de brouillard épais, les pêcheurs s'apprêtent à partir pour la pêche au merlan. Le groupe, dans des bateaux différents, suit la direction du chef, Jean-Baptiste à Isidore à petit Louis qu'on appelle ainsi pour le distinguer des 50 autres Jean-Baptiste Thibodeaux du village. Tout est prêt. Un moment de silence puis tous font le signe de la croix, ceux des bateaux et les autres du rivage. Jean-Baptiste commence les litanies. Les premiers Ora pro nobis se répercutent des bateaux au rivage et du rivage aux bateaux. Vers la fin de la journée, ils décident de manger et de se trouver un abri pour la nuit. En reprenant les rames, Jean-Baptiste reprend les litanies et il entend des voix autres que celles de ses hommes. Quand ils arrivent à la grève, ils sont face à face avec d'autres pêcheurs de leur village qui s'apprêtent à partir. Ils se trouvent donc au même endroit d'où ils sont partis le matin. Il va sans dire que Jean-Baptiste a dû subir des taquineries dont il se défendait en disant : "Quoi faire dans la brume"?

Ce que nous pouvons tirer de ces exemples, c'est que les Acadiens pratiquaient leur religion au meilleur de leur connaissance. Leur foi naïve et spontanée attendait tout de Dieu par l'entremise de son prêtre. Étant donné que le Dieu d'autrefois était surtout présenté comme Celui qui punit, il était inévitable que la foi en soit une de crainte. En somme, la crainte est le commencement de la sagesse .... Cette crainte n'aurait-elle pas contribué à la sagesse que nous découvrons chez la plupart de nos vieillards? En général, nos ancêtres se servaient de leur gros bon sens pour mener une vie pleinement heureuse remplie de joie, entremêlée de peines. Mais ils savaient vivre pleinement : travailler fort et s'amuser ferme.

Soeur Catherine Jolicoeur, f.m.a.

Centre Universitaire

St-Louis-Maillet, Edmundston, N.-B. 


\section{* LES SOURCES ORALES}

\begin{tabular}{|c|c|c|c|}
\hline AUDETTE, $\mathbf{M}^{\text {me }}$ Pierre & n. 1907 & Maltais & (Restigouche) N.-B. \\
\hline BOUlay, Honoré & n. 1895 & Balmoral & (Restigouche) N.-B. \\
\hline CARRIER, Bill & n. 1916 & Lorne & (Restigouche) N.-B. \\
\hline CARRIER, David & n. 1902 & Lorne & (Restigouche) N.-B. \\
\hline CARRIER, Estelle & n. 1893 & Lorne & (Restigouche) N.-B. \\
\hline CARRIER, Robert & n. 1899 & Lorne & (Restigouche) N.-B. \\
\hline Dolcet, Mme Wilfred & n. 1909 & Lorne & (Restigouche) N.-B. \\
\hline FrancoEur. Tom & n. 1895 & Lorne & (Restigouche) N.-B. \\
\hline GoDIN. Amanda & n. 1920 & Lorne & (Restigouche) N.-B. \\
\hline GOGUEN, Robert & n. 1893 & Cocagne & (Kent) N.-B. \\
\hline Guimond. Robert & n. 1923 & St-Quentin & (Restigouche) N.-B. \\
\hline LEBLANC, $\mathbf{M}^{\text {mee }}$ Irénée & n. 1895 & St-Joseph & (Westmorland) N.-B \\
\hline PARKER. Auzivina & n. 1930 & Dundee & (Restigouche) N.-B. \\
\hline ROBICHAUD, François & n. 1889 & Pointe-Sapin & (Kent) N.-B. \\
\hline
\end{tabular}

N.B. : L'Auteur de cet article a publié récemment. en 1981. à Montréal. chez Stanké. Les plus belles légendes acadiennes, $280 \mathrm{p}$. 